\title{
Lamellipod reconstruction by three dimensional reflection interference contrast nanoscopy (3D-RICN)
}

\author{
Marie-Julie Dejardin, ${ }^{\dagger}+$ Arnaud Hemmerle, ${ }^{\boldsymbol{\Phi}, \ddagger}$ Anaïs Sadoun, ${ }^{\dagger}$ Yannick Hamon, ${ }^{\S}$ Pierre-Henri \\ Puech, ${ }^{\dagger}$ Kheya Sengupta, ${ }^{,}, \boldsymbol{\Phi}$ and Laurent Limozin ${ }^{*} \dagger$ \\ $\dagger$ Aix-Marseille University, Inserm, CNRS, Adhesion $\mathcal{G}$ Inflammation, Marseille, France. \\ $\ddagger$ These authors contributed equally to this work \\ ฯAix Marseille University, CNRS, CINaM-UMR 7325, Marseille, 13288, France. \\ $\S$ Aix-Marseille University, CNRS, Inserm, CIML Marseille, France. \\ Received September 3, 2018; E-mail: sengupta@cinam.univ-mrs.fr; laurent.limozin@inserm.fr
}

\begin{abstract}
There are very few techniques to reconstruct the shape of a cell at nanometric resolution and those that exist are almost exclusively based on fluorescence, implying limitations due to staining constraints and artefacts. Reflection Interference Contrast Microscopy (RICM), a label-free technique, permits measurement of nanometric distances between refractive objects. However, its quantitative application to cells has been largely limited due to the complex interferometric pattern caused by multiple reflections on internal or thin structures like lamellipodia. Here we introduce three dimensional reflection interference contrast nanoscopy, 3D-RICN, which combines information from multiple illumination wavelengths and aperture angles to characterize the lamellipodial region of an adherent cell in terms of its distance from the surface and its thickness. We validate this new method by comparing data obtained on fixed cells imaged with Atomic Force Microscopy and Quantitative Phase Imaging. We show that as expected, cells adhering to micropatterns exhibit a radial symmetry for the lamellipodial thickness. We demonstrate that the substrate-lamellipod distance may be as high as $100 \mathrm{~nm}$. We also show how the method applies to living cells, opening the way for label-free dynamical study of cell structures with nanometric resolution.
\end{abstract}

Keywords: Super-resolution; Label-free imaging; Lamellipodium; Cell surface imaging; Interference imaging, Cell membrane topography.

Cell adhesion and spreading are central phenomena regulating cell function and behavior, ranging from activation to proliferation. ${ }^{1}$ Cell spreading, as well as motility, is usually mediated by a flat and dynamic protrusion, called the lamellipodium, ${ }^{2,3}$ which has been the object of numerous structural studies based on fluorescence ${ }^{4-6}$ and electronic microscopy. ${ }^{7}$ The shape of a motile cell, especially the lamellipodium, is known to be crucial for spreading, migration and mechanosensing. ${ }^{3,8,9}$ However, in cell adhesion studies the focus is often on the substrate-proximal surface, which is typically imaged using fluorescence based techniques to reveal specific proteins and structures involved in adhesion and associated signaling. ${ }^{10}$

An important aspect of adhesion is the quantification of the intimate contact of the cell membrane with the surface in terms of the cell to surface distance, usually achieved using different types of interference microscopy, ${ }^{11,12}$ allowing determination of distances between cell surfaces well below the optical resolution imposed by the limit of diffraction, out of reach for classical or confocal fluorescence microscosopy.
Different fluorescence related strategies, based on superresolution or deconvolution, and combined with total internal reflection fluorescence microscopy or dual objective astigmatism, can be also used to provide nanometer resolution in the axial direction. ${ }^{5,13-17}$ While permitting molecular specificity, fluo-based methods are limited by the necessity of labeling the sample, which is often inappropriate for fragile cells or medical samples. They are compromised by possible labeling or fixation artifacts ${ }^{18}$ and by photobleaching in case of long-term live or repeated observation.

In the context of quantification of adhesion, Reflection Interference Contrast Microscopy (RICM) has often been the technique of choice. ${ }^{11,19-21}$ It consists in epi-illuminating the cell-surface region with a monochromatic light and collecting the interfering rays. This purely interferometric approach, showed great initial promise, ${ }^{11,19}$ but was later mostly used in a semi-quantitative manner for cell analysis due to the complex optics of cells, though RICM does provide excellent resolution in the context of simple objects like colloids, model membranes or thin films. ${ }^{20-23}$ Recent application to cells of interferometric scattering microscopy (iS$\mathrm{CAT}^{24}$, which relies on scattering from fine features and edges, provides semi-quantitative informations at high time and lateral resolution, but with limited confidence in the nature of the structure imaged and limited resolution in vertical direction. ${ }^{25}$ Label-free techniques are currently undergoing a strong development, in particular to meet the needs of biomedical research, ${ }^{26,27}$ a striking example being the trawling of big databases of transmitted light images of cells using machine learning strategies. ${ }^{18,28}$ RICM of cells has known a revival in this context. ${ }^{29-31}$

RICM is sensitive to abrupt changes in the refractive index at the interface of two media, and in a first approximation, the collected intensity is related to the cell-surface distance. ${ }^{32-36}$ This approach was exploited to estimate membrane fluctuations. Two major problems associated with quantification of RICM images from cells are that the cell refractive index is not known and that extraneous reflections from internal organelles or the dorsal membrane may corrupt the data and render interpretation difficult. ${ }^{21,37}$ However, reflections from the dorsal membrane can provide an insight into the three dimensional shape of the lamellipod. To quantify this, we get inspiration from previous work that circumvented other limitations of RICM: multiple reflections which occur in presence of more than one interface, was overcome using a Fresnel model of multiple interfaces; ${ }^{22,38-40}$ and ambiguity due to the periodic relation between distance and phase shift, was overcome by dual wavelength illumina- 


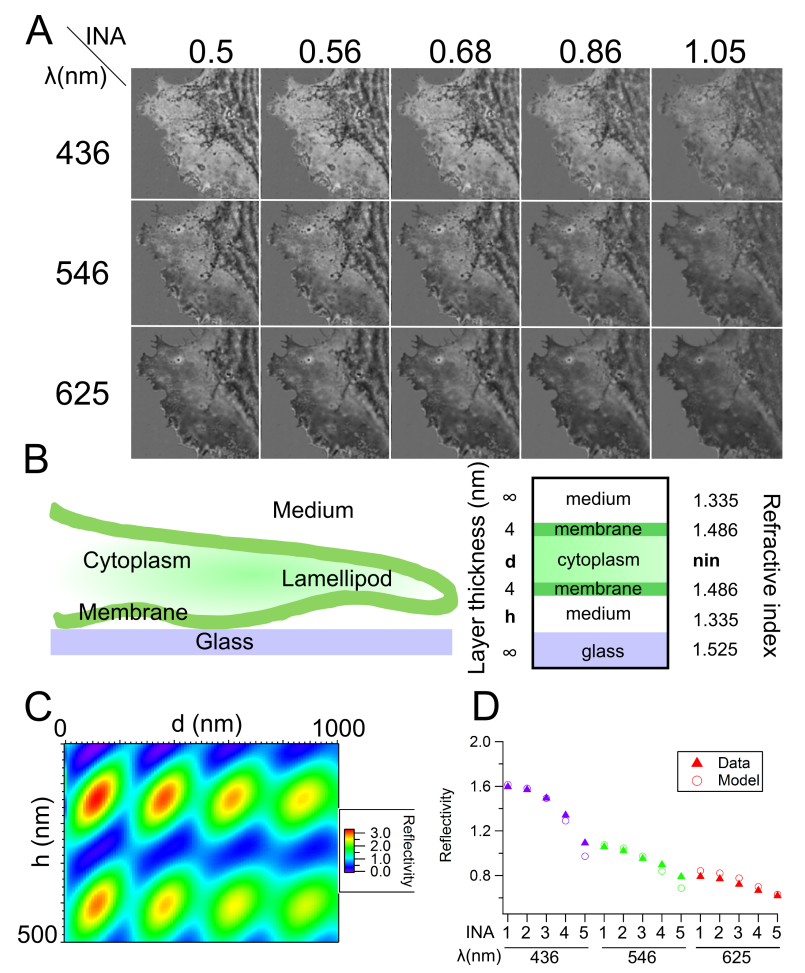

Figure 1. Principle of $3 \mathrm{D}-\mathrm{RICN}$. A. Montage of a multichannel reflectivity image stack. RICM images are taken sequentially with 3 illumination wavelengths $(\lambda)$ and 5 illumination numerical apertures (INA). Image background intensity is normalized to 1 , so that cell reflectivity is measured with respect to the background. The width of each image is ca $30 \mu \mathrm{m}$. B. Thin film model to calculate the reflectivity of a lamellipod with 3 parameters to determine: cell surface distance $h$, cytoplasm thickness $d$, cytoplasmic refractive index $n_{i n}$. C. Example of calculated reflectivity map ( $\left.\lambda=436 \mathrm{~nm}, I N A=0.95, n_{i n}=1.40\right)$ for variable cell surface distance $h$ and lamellipod thickness $d$. D. Example of measured reflectivity for a pixel taken in the middle of images A (triangle) and fitted theoretical reflectivity (circle) for each illumination condition (INA and wavelength).

tion. ${ }^{41,42}$ Here we image adherent cells in RICM mode, with multiple illumination angles and wavelengths, and develop a fitting procedure to reconstruct the three dimensional profile of the adhering lamellipodium with nano-metric resolution (3D-RICN).

To establish the proof of the principle, we chose the fibroblast-like simian cell-line COS-7, which spreads easily on uncoated glass, making large lamellipods and having relatively slow dynamics post-spreading. Cells were allowed to spread on glass and, after fixation, were imaged in RICM mode with multiple illumination conditions, obtaining 15 snapshots corresponding to combination of illumination wavelengths $436 \mathrm{~nm}, 546 \mathrm{~nm}$, and $625 \mathrm{~nm}$, and illumination numerical apertures (INA) of $0.5,0.56,0.68,0.86,1.05$ (Fig. 1A). Reflection of light from the sample is modeled assuming locally flat films oriented orthogonally to the optical axis, taking into account the $s$ and $p$ polarisation of light through the polarizer / quarter waveplate / analyzer, and using the Jones formalism to calculate the reflectivity taking into account multiple reflections and polarization $22,39,40$ (see SI for details). The lamellipodial region is modeled as a thin layer of cytoplasm of thickness $d$ and refractive index $n_{\text {in }}$, sandwiched between two membranes, the whole placed above the glass surface at a distance of $h$ (Fig. 1B). The images are normalized with respect to the background intensity, for comparing with theoretically generated map of the theoretical normalized reflectivity defined as $r=I_{\text {lam }} / I_{\mathrm{bgd}}$ with intensity $I_{\text {lam }}$ calculated for the lamellipod and $I_{\mathrm{bgd}}$ calculated for the background. For each experimental value of $\lambda$, INA and for variable $n_{\text {in }}$ (in 0.005 steps), a reflectivity map is generated for $h$ between 0 and $500 \mathrm{~nm}$ in $2 \mathrm{~nm}$ steps and $d$ between 0 and $1000 \mathrm{~nm}$ in $10 \mathrm{~nm}$ steps. Qualitatively, it accounts for an expected resolution better in $h$ than $d$ as well as a limit of sensitivity for large $h$ and $d$. An example of such a map for $\lambda=436 \mathrm{~nm}, \mathrm{INA}=0.95$ and $n_{\text {in }}=1.40$ is shown on fig. $1 \mathrm{C}$.

To solve the inverse problem of finding parameters $h, d$ and $n_{\text {in }}$ from the normalized multichannel data stack, a fitting error $\chi^{2}$ is calculated for each pixel of the experimental image (see SI), and the best solution $\left(h, d, n_{\text {in }}\right)$, corresponding to the minimal value of $\chi^{2}$, is identified. A graphical example of data and best-fit theoretical reflectivities for one randomly chosen pixel is shown on fig. $1 D^{*}$. Estimates of the error in $h, d$ and $n_{\text {in }}$ are expressed, for each pixel, in terms of the confidence interval $\delta h, \delta d$ and $\delta n_{i n}$, which quantifies the error introduced in fitting due to acquisition noise (see Fig. S2). The reconstruction algorithm was applied on an area of ca. $30 \mu \mathrm{m} \times 30 \mu \mathrm{m}$ chosen in the lamellipodial region.

The map of height $h$ and its error $\delta h$ are shown on Fig 2A,B. The distribution of $h$ is represented on the histogram $2 \mathrm{C}$, which exhibits a peak at $90 \mathrm{~nm}$. The thickness of the lamellipodium is described by the map of $d$ (Fig. 2D) and confidence interval $\delta d$ (Fig. $2 \mathrm{E}$ ). It is seen to be rather flat with the distribution of $d$ peaking at $75 \mathrm{~nm}$ (Fig. 2F). The map of cytoplasmic refractive index $n_{\text {in }}$ and its interval of confidence $\delta n_{\text {in }}$ are shown on Fig $2 \mathrm{G}$ and Fig $2 \mathrm{H}$. The distribution of $n_{i n}$, shown in the histogram of Fig 2J, peaks at 1.355. 3D surface plots of the basal and apical membranes were produced using $\mathrm{Fiji},{ }^{43}$ and are represented on Fig. 2K,L,M. Sequences with azimuthal rotation are available in Supp. Mat. The correlation between the fit parameters and their errors as well as the independence of the three fitted parameters are mapped in Fig. S4. Noticeably, $h$ and $d$ exhibit a certain degree of correlation shown in Fig. S4. Correlation is also visible between $d$ and $n_{\text {in }}$. This indicates that for certain pixel, there is an underdetermination of the 3 parameters.

Since no other technique is able to image a lamellipodium in $3 \mathrm{D}$ and with the accuracy reported here, to validate 3DRICN, we compared the reconstruction of the dorsal surface with an atomic force microscopy (AFM) image, and the optical thickness (expressed in terms of the optical path density) with quantitative phase image (QPI). The lamellipodial zone of the AFM image was oriented and scaled to correspond pixel by pixel to the 3D-RICN images, and was corrected for indentation of $40 \mathrm{~nm}^{\dagger}$ (Fig. 3A, raw image in Fig. S6) from which the apical membrane height $(\mathrm{AMH})$ was calculated by summing $h$ and $d$ (Fig. 3B). Comparison of $\mathrm{AMH}$ images $3 \mathrm{~A}$ and $3 \mathrm{~B}$ is provided on the density map of Fig. 3C, which exhibits a high density along the $x=y$ line, indicating a high overlap. Some points on the $y$ axis reflects errors due to incorrectly identified fringes in RICM. The profiles of $\mathrm{AMH}$ along the blue line of Fig. 3A and B are compared in Fig. 3D

${ }^{*}$ The code used for reconstruction is available and explained in details online: github.com/ArnaudHemmerle/3DRICN.

${ }^{\dagger}$ This is close to the typical deformation of $70 \mathrm{~nm}$ measured with AFM, see Fig. S5, which can arise from compression of the cytoplasm and/or pushing of the lower membrane against the substrate while imaging, even at a moderate force. 

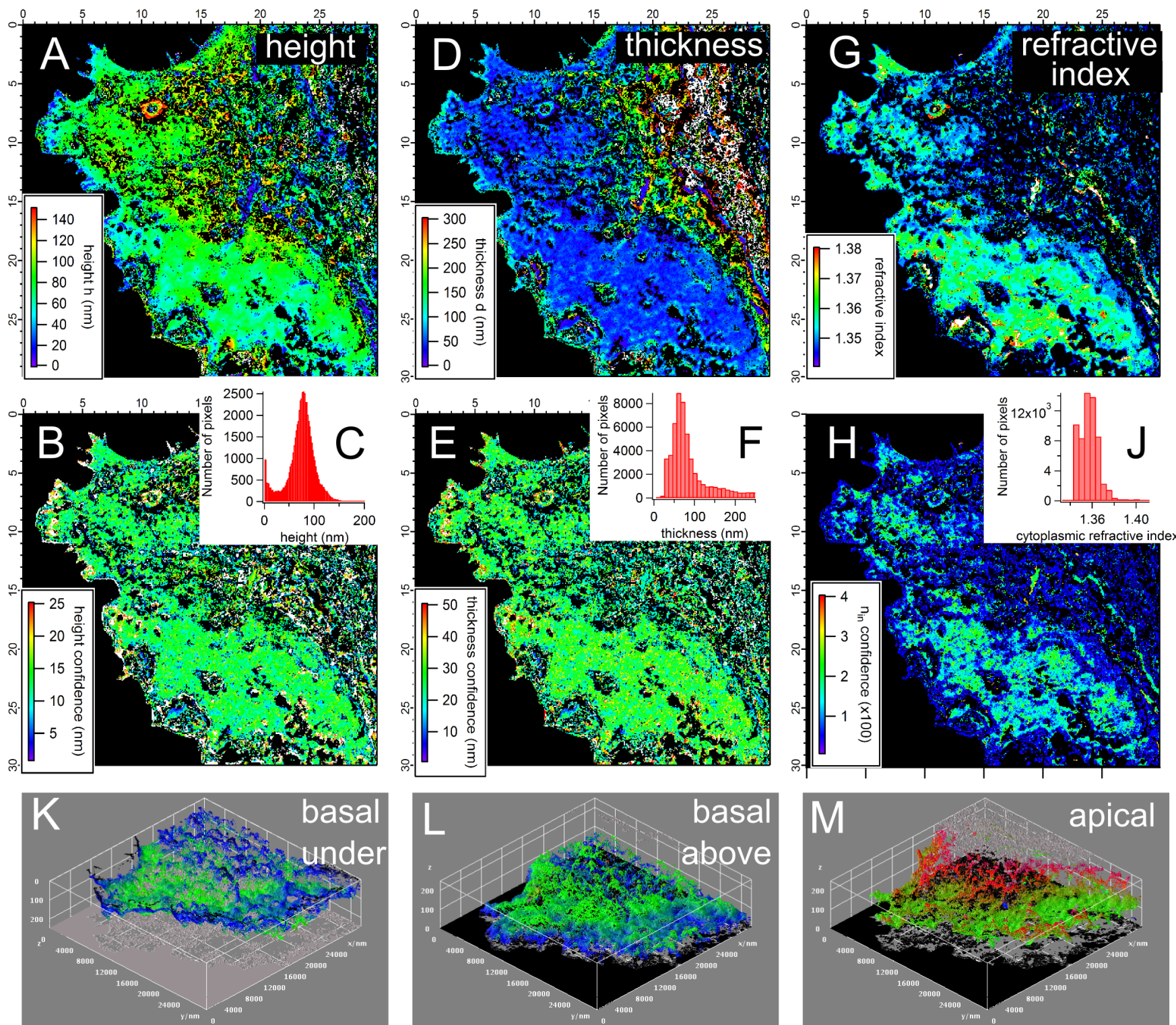

Figure 2. Topography obtained from the reconstruction algorithm. A. Map of height $h$ (cell-surface distance). B. Map of $\delta h$, confidence interval for $h$. C. Distribution of height $h$ on the entire image but excluding black pixels. D. Map of cell thickness $d$. E. Map of $\delta d$, confidence interval for $d$. F. Distribution of thickness $d$. G. Map of cytoplasmic refractive index $n_{\text {in }}$. H. Map of confidence interval for $n_{\text {in. }}$. J. Distribution of $n_{\text {in }}$. Note that only pixels that are deemed to be non-pathological (see SI) and correspond to $d>500 \mathrm{~nm}$ are represented. K, L, M: 3D representations of basal membrane (K: seen from under or L: seen from above) and of apical membrane (M:seen from top). Numbers on images axis indicate distance in $\mu \mathrm{m}$ for $2 \mathrm{D}$ maps $\mathrm{A}, \mathrm{B}, \mathrm{D}, \mathrm{E}, \mathrm{G}, \mathrm{H}$ and in $\mathrm{nm}$ for $3 \mathrm{D}$ reconstructions $\mathrm{K}$, $\mathrm{L}, \mathrm{M}$.

and show a good agreement. The standard height difference of the two profiles is less that $15 \mathrm{~nm}$, while the lateral difference is of the order of $100 \mathrm{~nm}$. A second test of 3D-RICN reconstruction was performed by comparison with Quantitative Phase Imaging (QPI). The same cell was imaged with QPI (Fig. 3E) and the zone of the lamellipod was croped, scaled and oriented to coincide pixel by pixel with 3D-RICN images. The optical path difference (OPD) was calculated from 3D-RICN data as $\mathrm{OPD}=\left(n_{\text {in }}-n_{\text {out }}\right) \times d($ Fig. S7A $)$. The OPD from QPI and 3D-RICN were compared as density maps (Fig. S7B) revealing a relatively poor agreement, likely because $n_{\text {in }}$ may be error-prone, as already suggested by the observed correlation between $d$ and $n_{\text {in }}$ identified in Fig. S4. We therefore corrected OPD from 3D-RICN taking a uniform value of $n_{\text {in }}=1.355$, which corresponds to the peak of $n_{\text {in }}$ distribution (Fig. 2J). This corrected OPD is mapped in Fig. 3F. The comparison is shown as a profile on Fig. 3G and a density map on Fig. 3H. This shows a fair agreement between the two methods. However the comparison is impeded by the observed limit of sensitivity of QPI in the lamellipod region, as well as inhomogeneous background level correction.

We next imaged a cell spread on a circular patch of diameter $65 \mu \mathrm{m}$, coated with the extracellular protein fibronectin. In this case, the normalization of the 3D-RICN raw images was performed by dividing the cell image by the aligned image of an empty fibronectin patch. The reconstruction was performed as described above for the non-patterned substrates. Results are shown on Fig. ??. The central region of the cell has been masked since the proposed reconstruction does not apply in this zone due to the presence of the nuclear membrane just above the proximal cell membrane. 

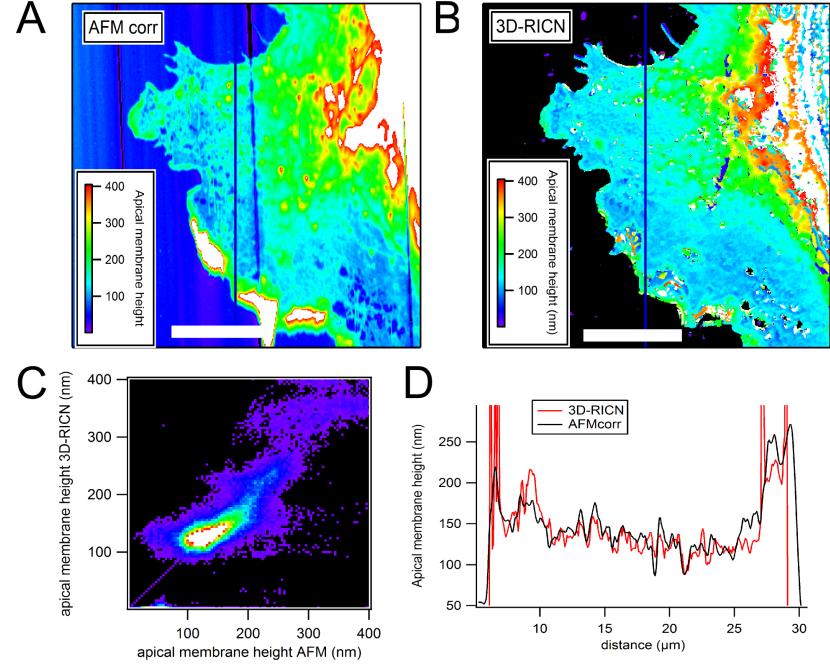

E
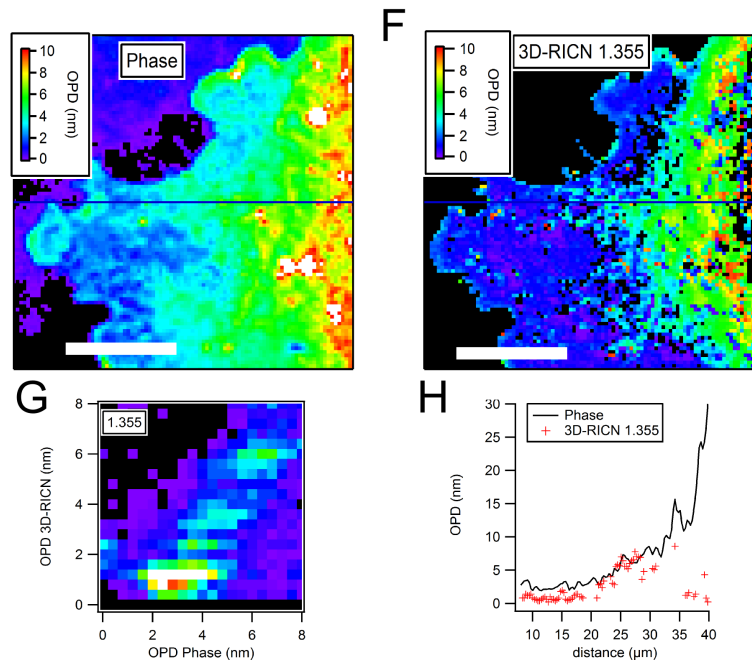

Figure 3. Comparison of 3D-RICN with AFM and QPI. A. AFM image of height of the apical membrane after correction for an average indentation of $40 \mathrm{~nm}$. B. 3D-RICN map of the apical membrane height, obtained as the sum $h+d$. C. Map density of the 3D-RICN map as a function of the corrected AFM image. D. Height profile taken along the vertical blue line in images $\mathrm{A}$, and B. E. OPD obtained by QPI. F. OPD obtained with 3D-RICN using $d$ and $n_{i n}=1.355$. G. Map density of OPD from F vs OPD from E. H. Profiles comparing OPD from $\mathrm{E}$ and $\mathrm{F}$ taken along the horizontal blue line. Scale bar for A, B, E, F: $10 \mu \mathrm{m}$.

Furthermore, the height of the apical membrane is expected to exceed $1 \mu \mathrm{m}$ where the model is not applicable. $h$ distribution peaks at $35 \mathrm{~nm}$ (Fig. ??C), much lower than in absence of fibronectin on the surface, with a globally uniform distribution (Fig. ??A). This is roughly consistent with integrin complex size. The histogram of cytoplasmic refractive index peaks at 1.355 (Fig. ??D), as was the case before. The cell thickness $d$ histogram peaks at $300 \mathrm{~nm}$ (Fig. ??E), and exhibits a radial distribution, as could be expected from the symmetry of the underlying pattern (Fig. ??B). The $\mathrm{AMH} / \mathrm{OPD}$ maps calculated from 3D-RICN data (Fig. ??F, resp. ??H) show good agreement with AFM/QPI (Fig. ??G, resp. ??J) (the latter assuming, as before, $n_{\text {in }}=1.355$ ).

We next applied the method to live cell imaging, reducing the acquisition time by a factor of 5 to make sure that it was short enough for the cell to be considered quasi-static during acquisition. This could be achieved by taking a single frame
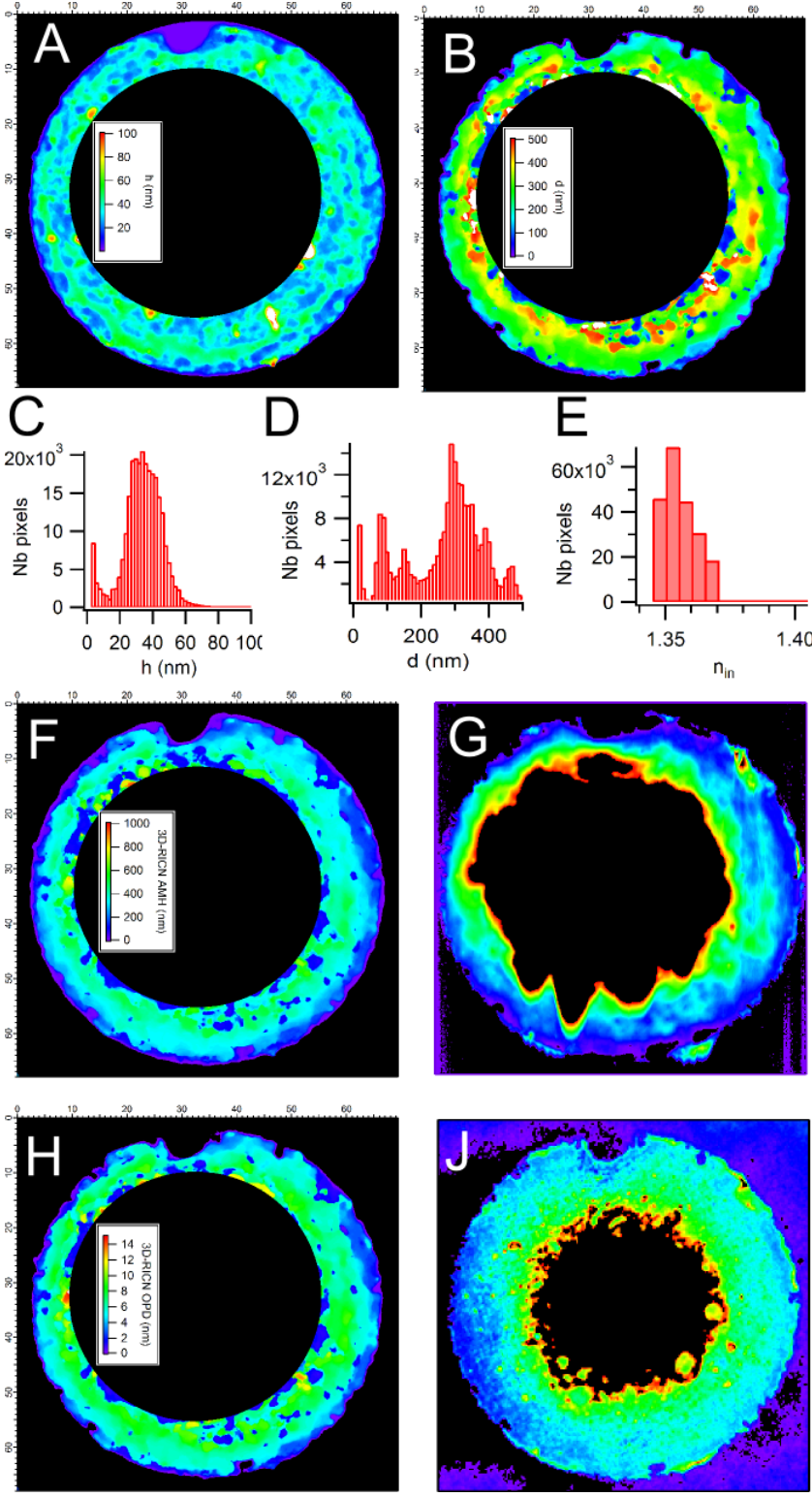

Figure 4. 3D-RICN reconstruction of a cell adhered on a patch of fibronectin and comparison with AFM and QPI. For clarity of the representation, the central part of the cell has been removed and a $0.8 \mu \mathrm{m}$ radius median filter has been applied on the $3 \mathrm{D}-\mathrm{RICN}$ reconstructions. A. Membrane height $h$. B. Cell thickness $d$. C Distribution of $h$. D. Distribution of cytoplasmic refractive index $n_{\text {in }}$. E. Distribution of $d$. F. Apical Membrane Height calculated from 3D-RICN data. G. AMH measured from AFM. H. Optical Path Distance calculated from 3D-RICN data. J. OPD measured from QPI. NB: Lateral scale for $\mathrm{A}, \mathrm{B}, \mathrm{F}, \mathrm{H}$ is in $\mu \mathrm{m}$.

in each channel, rather that 5 in the static reconstruction detailed before. The reconstruction of $h, d$ are shown on Fig. S9. A thinning of the cell (upper left corner, see arrow) upon retraction can be observed. The close contact of stress fibers is visible. Surprisingly, the extent of close contact is very limited, hinting at weak adhesion of the whole cell in the lamellipodial region. This confirms that the similar heights obtained on a fixed cell were not a mere artifact of fixation.

Future use of the technique for live imaging would require an accelerated acquisition. This could be realized by using a filter wheel and a piezo-mounted objective to adjust 
automatically the position of the focus depending on the wavelength, as well as a reduced set of INAs for imaging the cell. We have performed reconstructions of a close-up of the lamellipod for different sets of INAs to quantify how the $h$ and $d$ maps are influenced by the values and the number of INAs (Fig. S8). Taking the results obtained for the full set of five INAs as the reference, we first see that using only two INAs leads to wrong reconstruction of most pixels, in particular for $d$ and $h$. Taking three INAs largely improves the results, as shown also by the quantitative analysis of a patch of $40 \times 40$ pixels in an area of small roughness in the lamellipod. We conclude that the values of the INAs chosen have a strong influence on the quality of the reconstruction, and that taking extreme values for the INAs leads to a better reconstruction than taking closely spaced ones.

We also observe that the roughness of the patch, as defined by the Root Mean Square (rms) of height $h$, i.e. of the bottom membrane, is a good indicator for the quality of the reconstruction. Indeed, a wrong reconstruction of a pixel often leads to the convergence of the fit to extreme values far from the correct ones, leading to an artificial roughness of the image. Through a careful analysis, we determined that the set of 3 INAs [0.5, 0.68, 1.05], taking the two extreme values of the full set and an intermediate one, gives almost identical results as the reconstruction with the full set. This shows that a carefully chosen, smaller set of INA can be used in further studies for live imaging. Moreover, this convergence also ensures that our method is robust and uses enough INAs for a correct reconstruction of the lamellipod.

We also tested the reconstruction of the zones out of the lamellipod, including that under the nucleus, using appropriate layer-models. This however proved to be unsatisfactory, possibly because we were forced to limit the range of values searched due to the time required by the minimization algorithm. An obvious improvement will be the implementation of faster algorithms like progressive step refinement. One inherent problem of RICM that partly remains here is ambiguity linked to periodic fringes. Furthermore, the sensitivity of the technique to measure the refractive index is limited. Use of an additional wavelength may help to overcome both these problems. In addition to the reconstruction of the basal nuclear membrane, perspectives of applications for 3D-RICN include monitoring of apical membrane ruffle or mitochondrial membranes, as well as cell volume dynamics. Compared to widefield fluorescence, we can expect lower phototoxicity due to the alternance of illumination wavelengths in 3D-RICN.

To conclude, we have reconstructed the 3D shape of a lamellipod, with $10 \mathrm{~nm}$ axial resolution and $100 \mathrm{~nm}$ lateral resolution, of a label-free cell adhering to a functionalized substrate. We thus provide, for the first time to our knowledge, a validated reconstruction of the absolute height of the basal and apical membrane of a cell based on interference contrast microscopy principle. We have demonstrated the reconstruction of a live cell with time resolution of ca. one minute per frame. For both fixed and live cells, the lamellipod is relatively flat and with a thickness of about $100 \mathrm{~nm}$, as could have been expected from previous studies with AFM or with state-of-art super-resolution microscopy of the actin cytoskeleteton using double objective astigmatism. ${ }^{5}$ The substrate proximal surface, inaccessible to AFM, of a cell adhering non-specifically to glass was also imaged. The cell-surface distance was dis- tributed around $80 \mathrm{~nm}$ and reduced to about $40 \mathrm{~nm}$ when specific adhesion was present, which is compatible with fluorescence data for COS cells. ${ }^{5}$ Such ultra-fine topographical maps of the under-surface of cells are of interest not only for fundamental cell biophysics but also for applications like building cell-on-chip connections, for example in the context of neurotechnology. ${ }^{44}$ The method presented here opens the way to high throughput characterization of cell shape at nanometer resolution without labelling.

Acknowledgement We thank D. Marguet and S. Mailfert from the Picsel platform for lending of the Sid4bio camera, as well as S. Monneret for initiating us to quantitative phase measurement. We thank M. Biarnes-Pelicot and L. Borges (PMMC) for help with cell culture, O. Théodoly for stamps, and P. Dillard for preliminary experiments with Jurkat cells. PHP thanks JPK instruments for continuous support. This work was partially funded by GDR Imabio (MJD), AMIDEX (ANR-11-IDEX-0001-02) (AH and KS).

\section{Supporting Information Avail- able}

The file SI.pdf is available free of charge and contains figures and details on materials and methods.

\section{References}

(1) Cretel, E.; Touchard, D.; Bongrand, P.; Pierres, A. A new method for rapid detection of T lymphocyte decision to proliferate after encountering activating surfaces. J. Immunol. Meth. 2011, 364, 33-39.

(2) Giannone, G.; Dubin-Thaler, B. J.; Rossier, O.; Cai, Y. Chaga, O.; Jiang, G.; Beaver, W.; Döbereiner, H.-G.; Freund, Y.; Borisy, G.; Sheetz, M. P. Lamellipodial actin mechanically links myosin activity with adhesion-site formation. Cell 2007, 128, 561-575.

(3) Oakes, P. W.; Bidone, T. C.; Beckham, Y.; Skeeters, A. V. Ramirez-San Juan, G. R.; Winter, S. P.; Voth, G. A.; Gardel, M. L. Lamellipodium is a myosin-independent mechanosensor. Proc. Natl. Acad. Sci. U.S.A. 2018, 115, 26462651.

(4) Abraham, V. C.; Krishnamurthi, V.; Taylor, D. L.; Lanni, F. The actin-based nanomachine at the leading edge of migrating cells. Biophys. J. 1999, 77, 1721-1732.

(5) Xu, K.; Babcock, H. P.; Zhuang, X. Dual-objective STORM reveals three-dimensional filament organization in the actin $\mathrm{cy}^{-}$ toskeleton. Nat. Meth. 2012, 9, 185-188.

(6) Fritz-Laylin, L. K.; Riel-Mehan, M.; Chen, B.-C.; Lord, S. J. Goddard, T. D.; Ferrin, T. E.; Nicholson-Dykstra, S. M. Higgs, H.; Johnson, G. T.; Betzig, E.; Mullins, R. D. Actinbased protrusions of migrating neutrophils are intrinsically lamellar and facilitate direction changes. eLife 2017, 6 .

(7) Small, J. V.; Auinger, S.; Nemethova, M.; Koestler, S.; Goldie, K. N.; Hoenger, A.; Resch, G. P. Unravelling the structure of the lamellipodium. J. Microsc. 2008, 231, 479-485.

(8) Mogilner, A.; Keren, K. The shape of motile cells. Curr. Biol. 2009, 19, R762-R771.

(9) Dillard, P.; Varma, R.; Sengupta, K.; Limozin, L. Ligandmediated friction determines morphodynamics of spreading $\mathrm{T}$ cells. Biophys. J. 2014, 107, 2629-2638.

(10) Geiger, B.; Spatz, J. P.; Bershadsky, A. D. Environmental sensing through focal adhesions. Nat. Rev. Mol. Cell. Biol. 2009 10, 21-33.

(11) Curtis, A. S. The mechanism of adhesion of cells to glass. A study by interference reflection microscopy. J. Cell Biol. 1964, 20, 199-215.

(12) Paszek, M. J.; DuFort, C. C.; Rubashkin, M. G.; Davidson, M. W.; Thorn, K. S.; Liphardt, J. T.; Weaver, V. M. Scanning Angle Interference Microscopy Reveals Cell Dynamics at the Nano-scale. Nat. Meth. 2013, 9, 825-827.

(13) Sundd, P.; Gutierrez, E.; Pospieszalska, M. K.; Zhang, H. Groisman, A.; Ley, K. Quantitative dynamic footprinting microscopy reveals mechanisms of neutrophil rolling. Nat. Meth. 2010, $7,821-824$.

(14) Boulanger, J.; Gueudry, C.; Münch, D.; Cinquin, B.; PaulGilloteaux, P.; Bardin, S.; Guérin, C.; Senger, F.; Blanchoin, L.; Salamero, J. Fast high-resolution 3D total internal reflection fluorescence microscopy by incidence angle scanning and azimuthal 
averaging. Proc. Natl. Acad. Sci. U.S.A. 2014, 111, 1716417169.

(15) Bourg, N.; Mayet, C.; Dupuis, G.; Barroca, T.; Bon, P.; Lécart, S.; Fort, E.; Lévêque-Fort, S. Direct optical nanoscopy with axially localized detection. Nat. Photonics 2015, 9, 587.

(16) Brodovitch, A.; Limozin, L.; Bongrand, P.; Pierres, A. Use of TIRF to Monitor T-Lymphocyte Membrane Dynamics with Submicrometer and Subsecond Resolution. Cell. Molec. Bioengin. 2015, 8, 178-186.

(17) Cardoso Dos Santos, M.; Déturche, R.; Vézy, C.; Jaffiol, R. Topography of Cells Revealed by Variable-Angle Total Internal Reflection Fluorescence Microscopy. Biophys. J. 2016, 111, 1316-1327.

(18) Christiansen, E. M. et al. In Silico Labeling: Predicting Fluorescent Labels in Unlabeled Images. Cell 2018, 173, 792-803.e19.

(19) Bereiter-Hahn, J.; Fox, C. H.; Thorell, B. Quantitative reflection contrast microscopy of living cells. J. Cell Biol. 1979, 82, 767779 .

(20) Radler, J.; Sackmann, E. On The Measurement Of Weak Repulsive And Frictional Colloidal Forces By Reflection Interference Contrast Microscopy. Langmuir 1992, 8, 848-853.

21) Limozin, L.; Sengupta, K. Quantitative reflection interference contrast microscopy (RICM) in soft matter and cell adhesion. Chemphyschem 2009, 10, 2752-2768.

(22) Theodoly, O.; Huang, Z.-H.; Valignat, M.-P. New modeling of reflection interference contrast microscopy including polarization and numerical aperture effects: application to nanometric distance measurements and object profile reconstruction. Langmuir 2010, 26, 1940-1948.

(23) Huerre, A.; Jullien, M.-C.; Theodoly, O.; Valignat, M.-P. Absolute $3 \mathrm{D}$ reconstruction of thin films topography in microfluidic channels by interference reflection microscopy. Lab chip $\mathbf{2 0 1 6}$, 16, 911-916.

(24) Piliarik, M.; Sandoghdar, V. Direct optical sensing of single unlabelled proteins and super-resolution imaging of their binding sites. Nat. Comm. 2014, 5, 4495.

(25) Park, J.-S.; Lee, I.-B.; Moon, H.-M.; Joo, J.-H.; Kim, K.-H.; Hong, S.-C.; Cho, M. Label-free and live cell imaging by interferometric scattering microscopy. Chem. Sci. 2018, 9, 26902697.

(26) Bon, P.; Savatier, J.; Merlin, M.; Wattellier, B.; Monneret, S. Optical detection and measurement of living cell morphometric features with single-shot quantitative phase microscopy. $J$. Biomed. Optics 2012, 17, 076004 .

(27) Bon, P.; Barroca, T.; Lévêque-Fort, S.; Fort, E. Label-free evanescent microscopy for membrane nano-tomography in living cells. J. Biophotonics 2014, 7, 857-862.

(28) Pavillon, N.; Hobro, A. J.; Akira, S.; Smith, N. I. Noninvasive detection of macrophage activation with single-cell resolution through machine learning. Proc. Natl. Acad. Sci. U.S.A. 2018, 115, E2676-E2685.

(29) Lee, D.; Fong, K. P.; King, M. R.; Brass, L. F.; Hammer, D. A Differential dynamics of platelet contact and spreading. Biophys. J. 2012, 102, 472-482.

(30) Klein, K.; Maier, T.; Hirschfeld-Warneken, V. C.; Spatz, J. P. Marker-free phenotyping of tumor cells by fractal analysis of reflection interference contrast microscopy images. Nano Lett. 2013, 13, 5474-5479.

(31) Monzel, C.; Becker, A. S.; Saffrich, R.; Wuchter, P.; Eckstein, V.; Ho, A. D.; Tanaka, M. Dynamic cellular phynotyping defines specific mobilization mechanisms of human hematopoietic stem and progenitor cells induced by $\mathrm{SDF} 1 \alpha$ versus synthetic agents. Sci. Rep. 2018, 8, 1841.

(32) Pierres, A.; Eymeric, P.; Baloche, E.; Touchard, D.; Benoliel, A.-M.; Bongrand, P. Cell membrane alignment along adhesive surfaces: contribution of active and passive cell processes. Biophys. J. 2003, 84, 2058-2070.

(33) Zidovska, A.; Sackmann, E. Brownian motion of nucleated cell envelopes impedes adhesion. Phys. Rev. Lett. 2006, 96, 048103.

(34) Pierres, A.; Benoliel, A.-M.; Touchard, D.; Bongrand, P. How cells tiptoe on adhesive surfaces before sticking. Biophys. J. 2008, 94, 4114-4122.

(35) Mundinger, T. A.; Sommerfeld, A.; Reinehr, R.; Spatz, J. P. HÃd'ussinger, D.; Boehm, H. Investigating cell-ECM contact changes in response to hypoosmotic stimulation of hepatocytes in vivo with DW-RICM. P. One 2012, 7, e48100.

(36) Biswas, A.; Alex, A.; Sinha, B. Mapping cell membrane fluctuations reveals their active regulation and transient heterogeneities. Biophys. J. 2017, 113, 1768-1781.

(37) Sengupta, K.; Aranda-Espinoza, H.; Smith, L.; Janmey, P.; Hammer, D. Spreading of neutrophils: from activation to $\mathrm{mi}-$ gration. Biophys. J. 2006, 91, 4638-4648.

(38) Gingell, D.; Todd, I. Interference reflection microscopy. A quantitative theory for image interpretation and its application to cell-substratum separation measurement. Biophys. J. 1979, 26, $507-526$.

(39) Wiegand, G. Jaworek, T. Wegner, G. Sackmann, E. Studies of Structure and Local Wetting Properties on Heterogeneous, Micropatterned Solid Surfaces by Microinterferometry. J. Coll. Interf. Sci. 1997, 196, 299-312.

(40) Limozin, L.; Sengupta, K. Modulation of vesicle adhesion and spreading kinetics by hyaluronan cushions. Biophys. J. 2007, 93, 3300-3313.
(41) Schilling, J.; Sengupta, K.; Goennenwein, S.; Bausch, A. R. Sackmann, E. Absolute interfacial distance measurements by dual-wavelength reflection interference contrast microscopy. Phys. Rev. E 2004, 69, 021901.

(42) Sengupta, K.; Limozin, L. Adhesion of soft membranes controlled by tension and interfacial polymers. Phys. Rev. Lett. 2010, 104, 088101.

(43) Schindelin, J. et al. Fiji: an open-source platform for biologicalimage analysis. Nat. Meth. 2012, 9, 676-682.

(44) Toma, K.; Kano, H.; Offenhäusser, A. Label-free measurement of cell-electrode cleft gap distance with high spatial resolution surface plasmon microscopy. ACS Nano 2014, 8, 12612-12619. 
FOR TOC ONLY

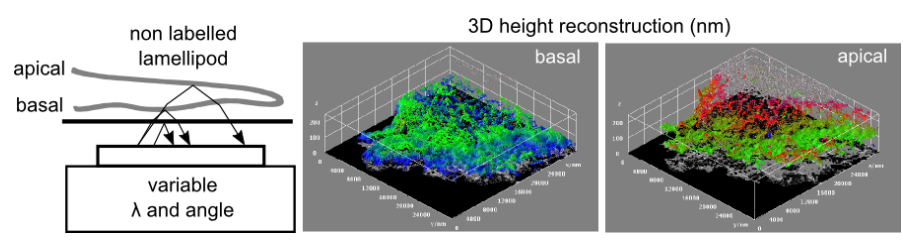




\section{Supplementary information}

Lamellipod reconstruction by three dimensional reflection interference contrast nanoscopy (3D-RICN)

Marie-Julie Dejardin 1*, Arnaud Hemmerle 2*, Anaïs Sadoun 1, Yannick Hamon 3, Pierre-Henri Puech 1, Kheya Sengupta $2 \dagger$, Laurent Limozin $1 \dagger$

1. Aix-Marseille University, Inserm, CNRS, Adhesion \& Inflammation, Marseille, France.

2. Aix Marseille University, CNRS, CINaM-UMR 7325, Marseille, 13288, France.

3. Aix-Marseille University, CNRS, Inserm, CIML Marseille, France.

* These authors contributed equally to this work.

$\dagger$ Corresponding authors: laurent.limozin@inserm.fr, sengupta@cinam.univ-mrs.fr

\section{Contents:}

- Material \& Methods:

- Sample preparation

- Optical setup

- Optical imaging

- Atomic force microscopy (AFM) imaging and force mapping

- Modeling 3D-RICN

- 3D-RICN image reconstruction

- Supplementary Movies 1-3.

- Supplementary Figures S1-S9. 


\section{Material \& Methods}

Sample preparation: Fibroblast-like cells of the COS-7 cell line were cultivated in DMEMc medium with $5 \% \mathrm{CO}_{2} .24 \mathrm{~h}$ before observation, cells were detached with 5\% EDTA, and were deposited in a petri dish with glass bottom (Fluorodish FD-35, $e=170 \mu \mathrm{m}, \mathrm{WPI})$ at $37^{\circ} \mathrm{C}$. Alternatively, the glass bottom was first coated by incubating during 10 min a $10: 1 \mathrm{mixture}$ of fibronectine $(50 \mu \mathrm{g} / \mathrm{ml}$, Sigma) and fluorescently labeled fibrinogen $(5 \mu \mathrm{g} / \mathrm{ml}$, Thermofisher).

Before some observations, cells were fixed with $2 \%$ paraformaldehyde during 15 min and rinsed with PBS. The microscope chamber was filled up with about $1 \mathrm{ml}$ of PBS, resulting in a liquid height of about $2 \mathrm{~mm}$ above the cells.

Patterning of the substrate with fibronectin was performed by using Micro-Contact Printing Method (Thery M and Piel M, Cold Spring Harb Protoc. 2009 Jul;2009(7):pdb.prot5255). PDMS stamp exposing disks of $65 \mu \mathrm{m}$ diameter were inked with a mixture of fibronectin and fluorescent fibrinogen for 45 minutes at room temperature in the dark. Then, the ink was removed and the PDMS stamp gently dried with $\mathrm{N}_{2}$. The stamp was transferred to the petri dish glass surface for 10 minutes at $37^{\circ} \mathrm{C} 5 \% \mathrm{CO}_{2}$ and then removed. The glass surface was extensively washed with PBS $1 \mathrm{X}$ and backfilled with pluronics F127 2\% (Sigma) for 1 hour at room temperature in the dark, in order to avoid non-specific adhesion.

Optical setup: The light source is a Lumen 200 (Prior) connected to the microscope via a liquid optical fiber. The inverted microscope AxioObserver (Zeiss) is equiped with: three passband filters (436/20, 546/10, 625/26 nm), protected with heat filters and disposed on a slider; a motorized aperture diaphragm; a fluorescence cube containing a polarizer, a $50 / 50$ semi reflector mirror and a crossed analyzer; an objective Antiflex $63 \mathrm{x}$ NA=1.25 devoided of phase ring (Carl Zeiss). Camera is a $1004 \times 1002$ pixels, 16 bits Andor iXon+. Optionally, an additional 1.6x magnification integrated to the microscope was used (Optovar). Andor Camera and microscope are controlled by MicroManager, allowing semi-automated image acquisition. For measurements with the phase camera Sid4Bio (Phasics, Palaiseau, France), a dual port (Carl Zeiss) is used to connect both cameras simultaneously to the sideport of the microscope. The phase camera was controlled by a dedicated computer using the Sid4Bio software (Phasics).

Optical imaging: For 3D-RICN imaging, image acquisition was performed by exposing the sample successively to green, blue and red light using the passband filters. The focus was carefully adjusted between each wavelength in order to compensate for chromatic aberrations of the objective. For each wavelength $\lambda$, five different openings of the aperture diaphragm were automatically and successively selected, corresponding to Illumination Numerical Apertures INA=0.5, 0.56, 0.68, 0.86, 1.05. The exposure time, typically comprised between 0.1 and $1 \mathrm{~s}$, was adapted in order to obtain in average about 3000 counts per pixel on the camera for each illumination $(\lambda$, INA). 5 images were taken and averaged at each illumination. Finally, raw RICM images of the cells were compiled in a test stack of 15 images corresponding to 15 ( $\lambda$, INA) combinations or channels. A reference stack was taken in identical conditions in a neighbouring region of the sample devoided of cells. Finally, the value of the camera offset was subtracted for test and reference stack, and a stack of images with background normalized to 1 was obtained using image by image division of the test stack by the reference stack. An example of normalized multichannel stack is shown on the montage of fig. 1A. For live cell imaging, only one frame was taken in each illumination. A calibration of the INAs was performed with a simpler system consisting in a glass shard forming an edge with the glass substrate in PBS (see Fig. S1).

For quantitative phase imaging, the halogen lamp was set to $7 \mathrm{~V}$ and the condenser for transmission bright field microscopy was adjusted for Köhler illumination. The recorded interferograms were transformed automatically in OPD images by the Sid4Bio software. A reference image was first taken in a cell free zone of the sample. Then a series of 5 images with exposure ca. $1 \mathrm{~s}$ each was taken and averaged to produce one phase image representing the map of optical thickness. ${ }^{26}$ In case of patterns, an empty (cell-free) motif was taken as reference.

Atomic force microscopy (AFM) imaging and force mapping: AFM was performed using a JPK Nanowizard 1 (z range $15 \mu \mathrm{m}$ ), mounted on an Zeiss Axiovert 200M. Gold coated MLCT levers (Veeco, nominal spring constant 10pN/nm) were used. Contact mode was used for imaging. Set point and gains were manually adjusted to keep the applied force the lower possible ie around $100 \mathrm{pN}$, while scanning at $0.3-0.5 \mathrm{~Hz}$ areas as large as $100 \mu \mathrm{m} \times 100 \mu \mathrm{m}$, and down to $15 \mu \mathrm{m} \times 15 \mu \mathrm{m}$. Areas of interest were chosen using transmision and/or fluorescence microscopy, in order to localize and find the exact same cells as the ones examined using other optical techniques performed on a different microscope. At the end of imaging, the cantilever was calibrated using built-in procedures based on the analysis of its thermal motion. Sensitivity was determined using contact with cell-free glass surface. This allowed us to determined that our imaging forces were as low as $100 \mathrm{pN}$ in regular conditions.

For force mapping, 16 pts x 16 pts grids were set onto the AFM imaged zone. Typical contact force was 100 to 150 $\mathrm{pN}$, indentation and retraction speeds were $1-2 \mu \mathrm{m} / \mathrm{s}$ for a motion range of up to $3 \mu \mathrm{m}$. Rather flat parts of the cells were selected in order to be able to gain complete force curves, regarding our $15 \mu \mathrm{m}$ piezo range. One force curve was collected per force pixel, and was analysed using a homemade python set of functions, allowing to fit the pressing curve, after tip-sample correction, with a linear model for the contact zone to estimate the indentation. Indentation maps were then rebuilt and an average indentation estimated, since the force pixels were, in order to keep the experiments doable and the data tractable, by far larger than the optical ones (Fig. S5) (Franz CM, Puech PH 2008 Cell. Molec. Bioengin. 1(4) :1865.)

Modeling 3D-RICN: Reflection of light from the sample is modeled assuming locally flat films oriented orthogonally to the optical axis. Taking into account the $s$ and $p$ polarisation of light through the polarizer / quarter waveplate / analyzer, the reflected intensity is equal to: ${ }^{22}$ 


$$
I=\frac{\alpha}{1-\cos \theta_{1}} \int_{0}^{\theta_{1}}\left|R_{s}-R_{p}\right|^{2} \sin \theta d \theta
$$

with INA $=n_{1} \sin \theta_{1}, \alpha$ a constant, and integration runs over illumination angle $\theta$.

Reflectivity can be calculated using Jones matrices formalism, taking into account multiple reflections and polarization: ${ }^{22,39,40}$

$$
M_{j}=\left(\begin{array}{cc}
\cos \beta_{j} & \frac{i \sin \beta_{j}}{N_{j}} \\
i N_{j} \sin \beta_{j} & \cos \beta_{j}
\end{array}\right)
$$

with $\beta_{j}=\frac{2 \pi d_{j}}{\lambda} n_{j} \cos \theta_{j}, \lambda$ the wavelength, $n_{j}$ and $d_{j}$ the refractive index and the thickness of layer $j, \theta_{j}$ the angle of incidence in layer $j$, and $N_{j}$ the effective index in layer $j$ defined as: $N_{j}=N_{j}^{s}=n_{j} / \cos \theta_{j}$ for $s$ polarization and $N_{j}=N_{j}^{p}=n_{j} \cos \theta_{j}$ for $p$ polarization

$$
\begin{gathered}
M=\prod_{j=1}^{m} M_{j}=\left(\begin{array}{ll}
m_{11} & m_{12} \\
m_{21} & m_{22}
\end{array}\right) \\
R_{s, p}=r_{0, m+1}^{s, p}=\frac{m_{11} N_{0}^{s, p}-m_{22} N_{m+1}^{s, p}+m_{12} N_{0}^{s, p} N_{m+1}^{s, p}-m_{21}}{m_{11} N_{0}^{s, p}+m_{22} N_{m+1}^{s, p}+m_{12} N_{0}^{s, p} N_{m+1}^{s, p}+m_{21}}
\end{gathered}
$$

In the case of the lamellipod, one considers for the model a thin layer of cytoplasm comprised between two membranes and hovering above the glass surface of the sample (Fig. 1B). The bottom membrane to glass distance is noted $h$, the lamellipod thickness $d$, with unknown refractive index $n_{\text {in }}$ comprised between 1.34 and 1.40 (fig. 1B). The theoretical normalized reflectivity is defined as $r=I_{\mathrm{lam}} / I_{\mathrm{bgd}}$ with the intensity $I_{\text {lam }}$ calculated for the lamellipod and $I_{\mathrm{bgd}}$ calculated for the background. For each experimental value of $\lambda$, INA and for variable $n_{\text {in }}$ (in 0.005 steps), a reflectivity map is generated for $h$ between 0 and $500 \mathrm{~nm}$ in $2 \mathrm{~nm}$ steps and $h$ between 0 and $1000 \mathrm{~nm}$ in $10 \mathrm{~nm}$ steps. Example of such a map for $\lambda=436 \mathrm{~nm}$, INA=0.95 and $n_{\text {in }}=1.40$ is shown on fig. $1 \mathrm{C}$.

3D-RICN image reconstruction The inverse problem of finding parameters $h, d$ and $n_{\text {in }}$ from the normalized multichannel data stack is performed as follows. For each pixel of the experimental image, a fitting error $\chi^{2}$ is calculated

$$
\chi^{2}\left(h, d, n_{\text {in }}\right)=\Sigma_{i} \frac{\left(I_{\text {simu }, \mathrm{i}}\left(h, d, n_{\text {in }}\right)-I_{\text {data }, \mathrm{i}}\right)^{2}}{I_{\text {simu }, i}\left(h, d, n_{\text {in }}\right)}
$$

where the sum is performed on i different illuminations. The best solution $\left(h, d, n_{\text {in }}\right)$ is obtained for the minimal value of $\chi^{2}$. A graphical example of data and theoretical reflectivities for one given pixel is shown on Fig. 1D.

The reconstruction algorithm was implemented in Python 3 and conceived to run on a multicore processor by division of the image in the same number of subimages as the number of cores. The strategy consists in comparing, for each pixel, the series of measured illuminations with a 3D model matrix calculated for $h, d$ and channel number. The coordinates of the minimum of the difference matrix, corresponding to the best parameters, are recorded. This procedure is looped for every value of the refractive index tested. The current implementation running on a 24 core i7 computer with 64 Go RAM, with operating system Windows 7 pro, would reconstruct an image of $1000^{*} 1000$ pixels in $1.5 \mathrm{~h}$, with 17 possible values for $n_{\text {in }}$, 2 $\mathrm{nm}$ step for $h, 10 \mathrm{~nm}$ step for $d$.

The detailed procedure for one example pixel is illustrated on Fig. S2. The $\chi^{2}$ map shows periodic valleys in the $(h, d)$ plane. To estimate a confidence interval associated with the best solution, the next solutions (corresponding to the values of $\left.\chi^{2}<\chi_{\max }^{2}\right)$ are also considered. $\chi_{\max }^{2}$ was chosen from a measure of the noise in data, obtained by reconstructing individually the 5 frames recorded before averaging. For each pixel, $\chi_{\max }^{2}$ is taken equal to the highest $\chi^{2}$ out of the 5 reconstructions. This sets a range of acceptable solutions $\left(h, d, n_{\text {in }}\right)$, with respect to the data noise, on which ensemble statistics are calculated, like the range, the median and the interquartile range. We note $\delta h, \delta d, \delta n_{\text {in }}$ respectively the interquartile range of $h, d, n_{\text {in }}$. Fig. S3 shows a comparison of reconstruction parameters between best solution and median solution.

To restrict the representation to the lamellipod in Fig. 2, only the pixels corresponding to a thickness between 1 and 500 $\mathrm{nm}$ are shown. Additionally, pixels were not represented when certain conditions, considered as pathological, were fulfilled: (a) $d>50$ and $\delta d<15 \mathrm{~nm}$, to prevent too low $\delta d$ above a minimal $d$; (b) $n_{\text {in }}<1.3475$ and $\delta n_{\text {in }}>0.0075$ to prevent too high $\delta n_{\text {in }}$ under a minimal $n_{\text {in }}$ (c) $h>50 \mathrm{~nm}$ and $d<15 \mathrm{~nm}$ to prevent low $d$ at high $h$.

Surface plots of basal and apical membranes (Fig. 2K,L,M) were produced using the 3D surface plot plugin for ImageJ implemented in Fiji. ${ }^{43}$ A preliminary median filter with a radius of 4 pixels was applied on images $2 \mathrm{~A}, \mathrm{D}$. 


\section{Supplementary Movies}

Supplementary Movie 1: Azimutal rotation of the 3D surface plot of the basal membrane seen from under, corresponding to Fig. 2K. Axis scale in nm.

Supplementary Movie 2: Azimutal rotation of the 3D surface plot of the basal membrane seen from above, corresponding to Fig. 2L. Axis scale in $\mathrm{nm}$.

Supplementary Movie 3: Azimutal rotation of the 3D surface plot of the apical membrane seen from above, corresponding to Fig. 2M. Axis scale in $\mathrm{nm}$. 
Supplementary Figures
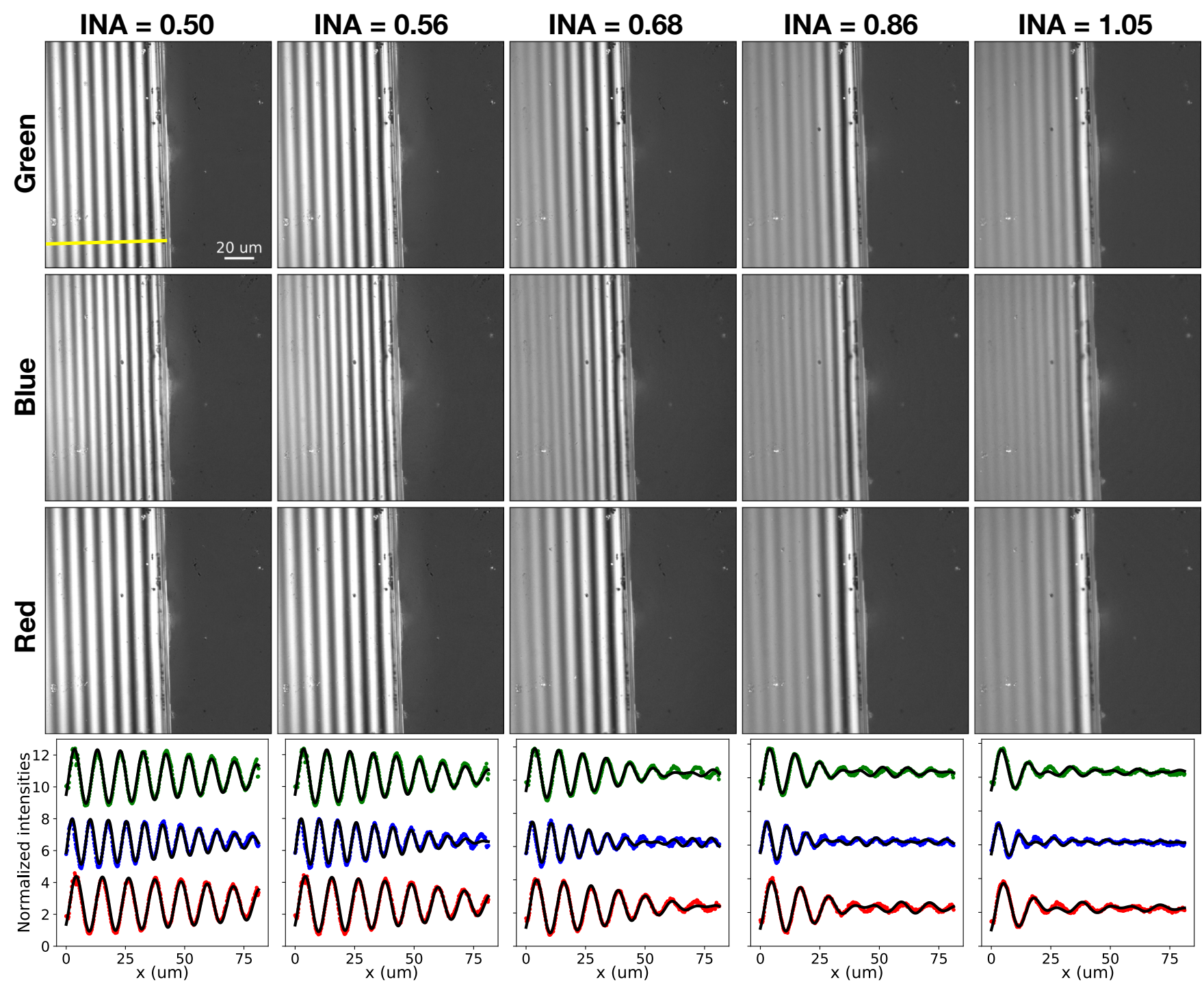

Figure S1. RICM images at various INA and wavelengths of a glass shard deposited in water on a glass substrate, forming an edge with an angle of 22 mrad. The bottom row shows fits of the intensity profiles on a line perpendicular to the fringes for the three colors simultaneously (shown on the top left image), allowing for a determination of each INA with a precision of \pm 0.02 . Blue and green profiles are shifted vertically for convenience. 

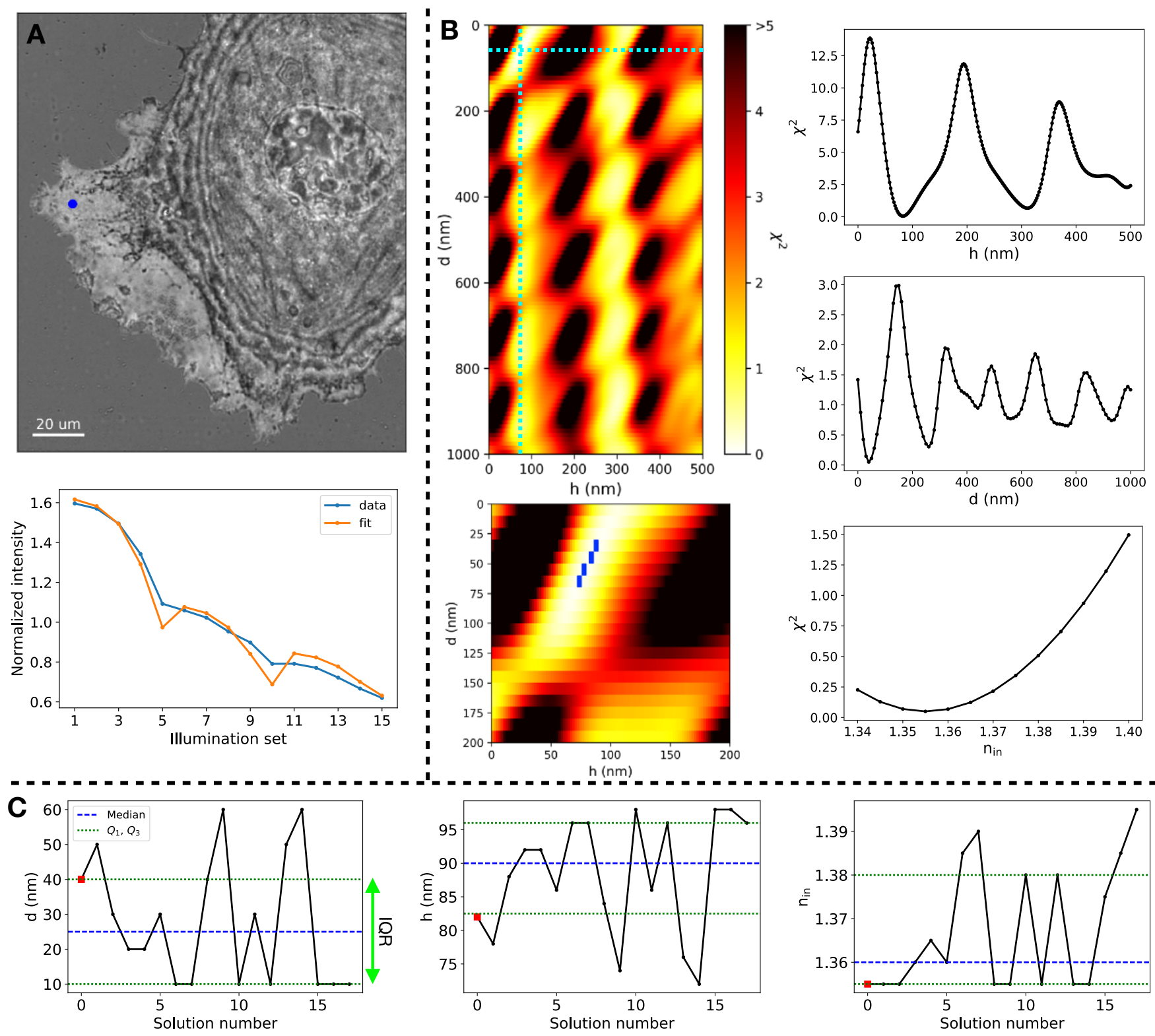

Figure S2. (a) Top: RICM image of the cell showing the position of the pixel of interest. Bottom: Normalized intensities for the pixel and associated best fit. (b) Top left: $\chi^{2}$ landscape of the pixel at a fixed $n_{\text {in }}$. Right: $\chi^{2}$ profiles following the dashed lines on the $\chi^{2}$ landscape, and as a function of $n_{\text {in }}$ for $h$ and $d$ fixed. Bottom left: zoom on the landscape around the best solution. The blue pixels at low $\chi^{2}$ correspond to solutions $\left(h, d, n_{\text {in }}\right)$ with $\chi^{2}<\chi_{\max }^{2}$ used for the analysis shown in (c). (c) Values of $d, h$ and $n_{\text {in }}$ for the solutions with $\chi^{2}<\chi_{\max }^{2}$. The red squares show the best value (smallest $\chi^{2}$ ), the blue dashed lines is the median, and the green lines are the first and third quartiles defining the interquartile range (IQR).
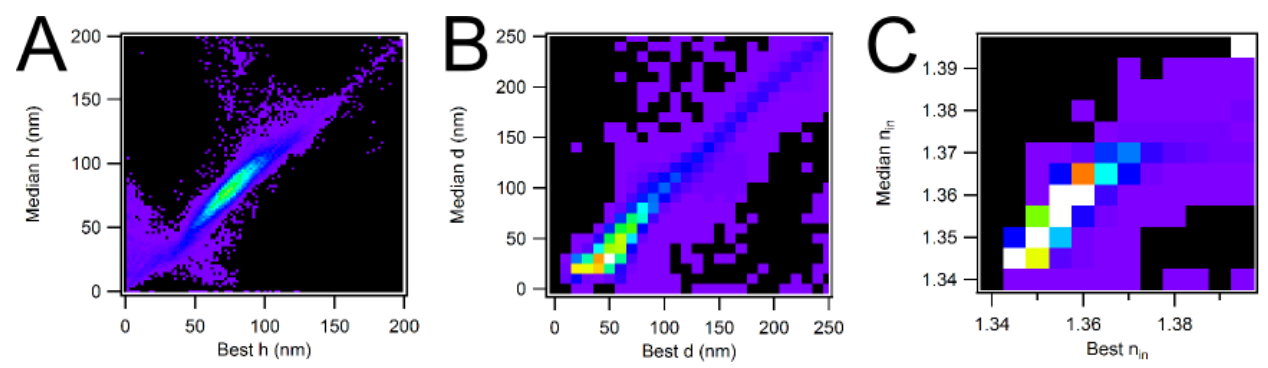

Figure S3. Comparison of different methods for determining $h, d$ and $n_{\text {in }}$. This demonstrates a fairly close agreement between the 2 solutions, except the median thickness which is significantly lower than the best thickness for $d<50 \mathrm{~nm}$ (Fig. S3B). 

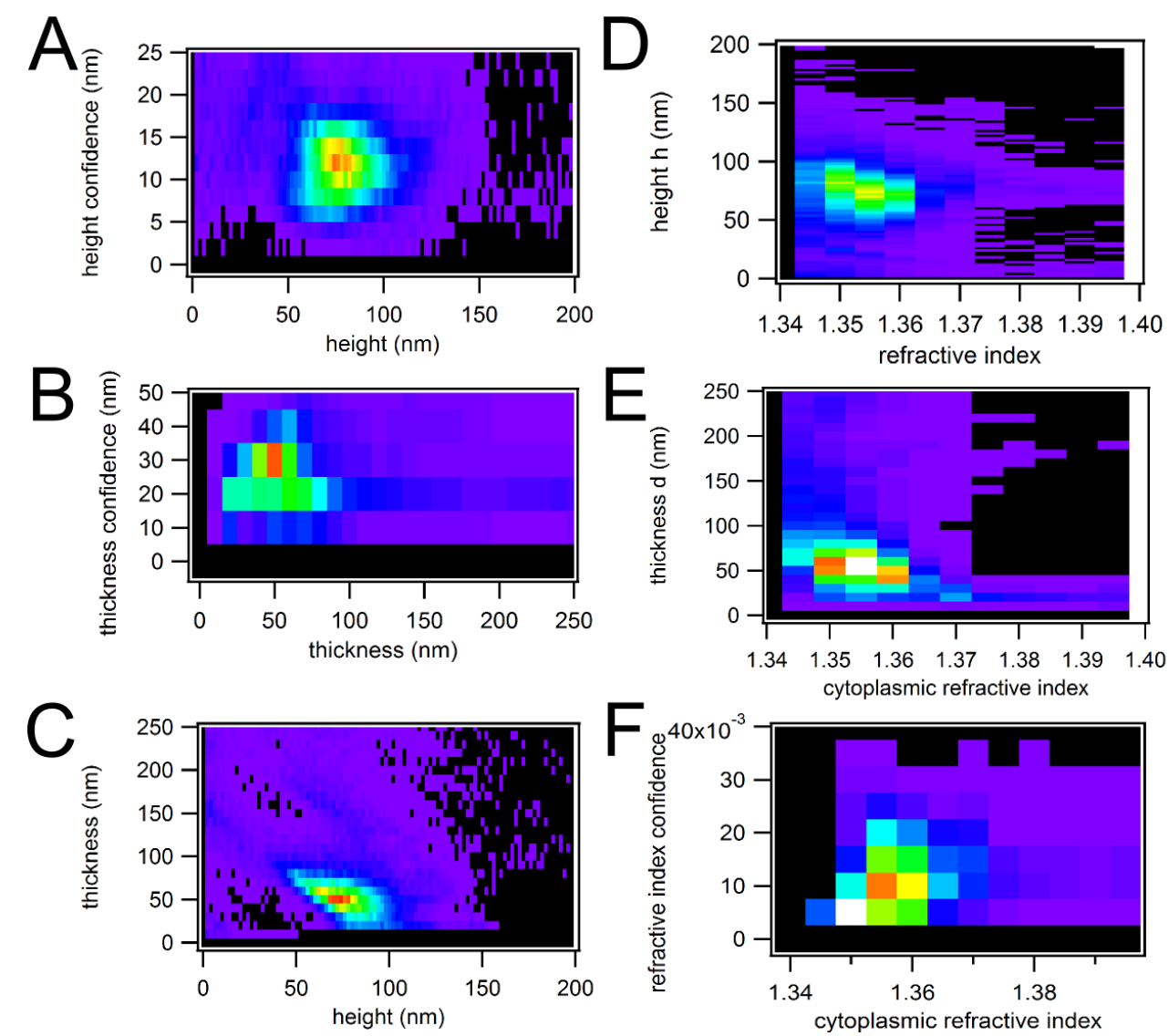

Figure S4. Density maps illustrating the degree of correlation between the parameters $h, d, n_{\text {in }}$ and their confidence interval. Data correspond to the reconstructions shown in Fig. 2. A. Density map of height $h$ and height confidence $\delta h$ showing the absence of correlation between these quantities. B. Relation between thickness $d$ and confidence interval $\delta h$. C. The density map shows a certain degree of correlation between height $h$ and thickness $d$. D-F. The dependence of $h, d$ and $\delta n_{i n}$ as a function of $n_{\text {in }}$. A certain degree of correlation between $d$ and $n_{\text {in }}$ is visible.
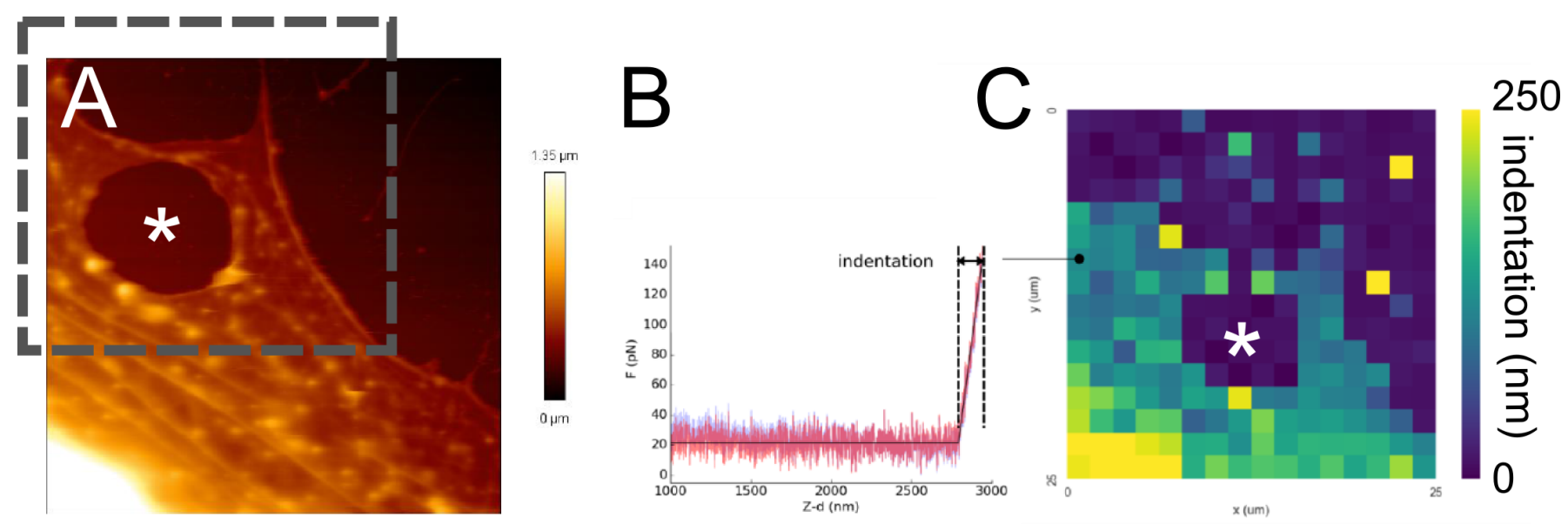

Figure S5. Indentation mapping in AFM. A. Part of a lamellipodium imaged at a force of $100 \mathrm{pN}$ in contact mode. B. Typical force curve obtained in this region, with the determination of the indentation at a force comparable as the force used for imaging. C. Reconstruction of the indentation map of a region of A, considering a particularly recognizable zone $\left(^{*}\right)$. 


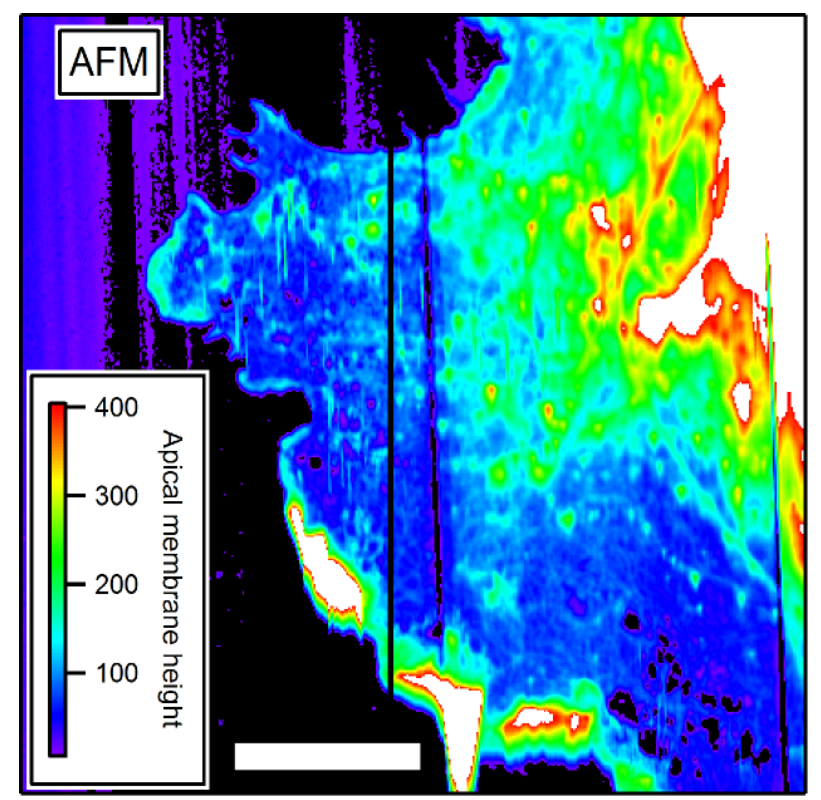

Figure S6. Complement to Main Fig.3. Raw AFM image of Fig. 3A. Scale bar: $10 \mu \mathrm{m}$.

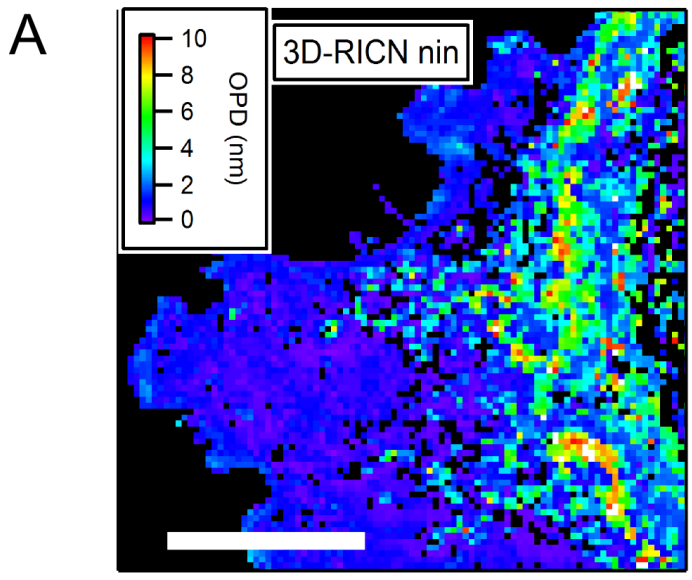

$B$

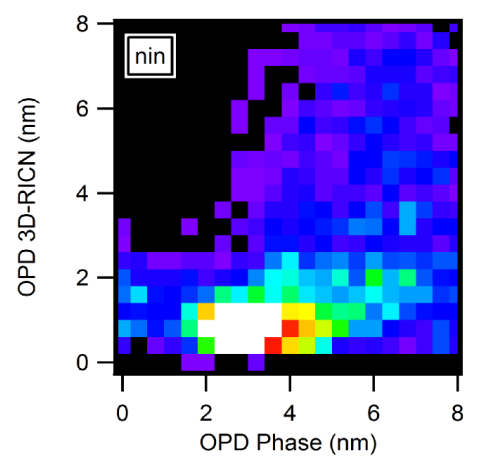

Figure S7. Complement to 3D-RICN QPI comparison (Main Fig.3). A. OPD obtained with 3D-RICN using $d$ and $n_{i n}$. B. Density map for image A and phase image Fig.3E. Scale bar: $10 \mu \mathrm{m}$. 
$[0.5,0.56,0.68$ $0.86,1.05]$

$[0.5,0.68,1.05] \quad[0.5,0.56,0.68]$

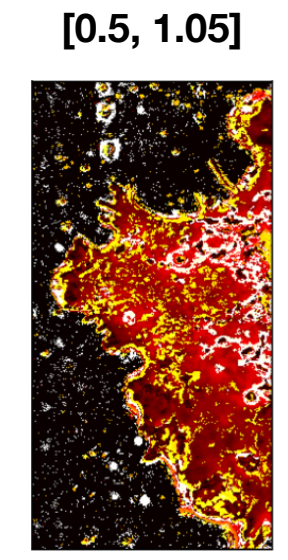

$[0.5,0.56]$
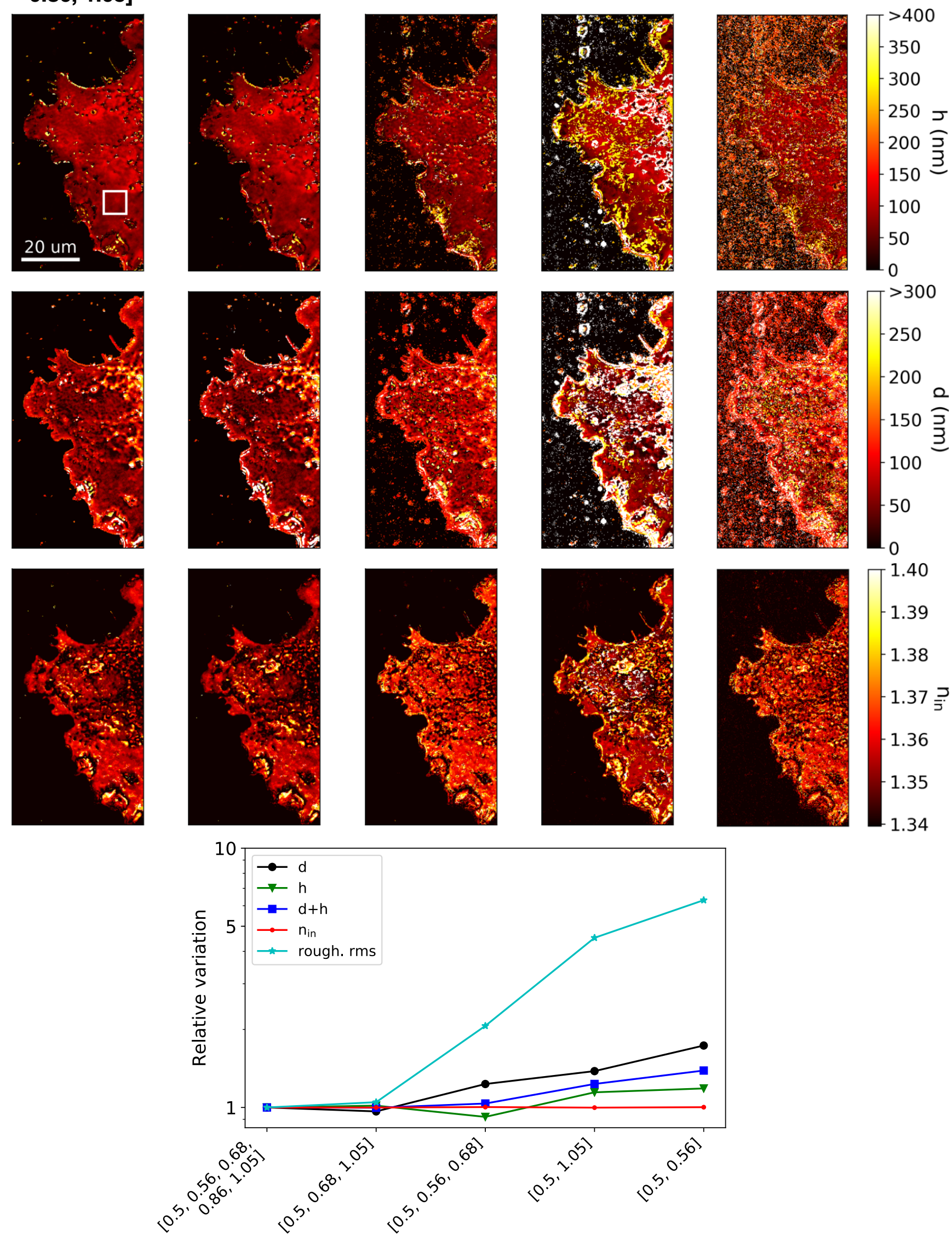

Figure S8. Top: Reconstructions of the lamellipod using the best solution of the model for different sets of INAs. Bottom: Analysis of the mean variation of $d, h, d+h, n_{\text {in }}$ and the roughness of the patch, as defined by the white square on the top left image. The variations are relative to the reconstruction obtained with the full set of INAs. The roughness is here defined as the Root Mean Square (rms) height of $h$ (bottom membrane). 


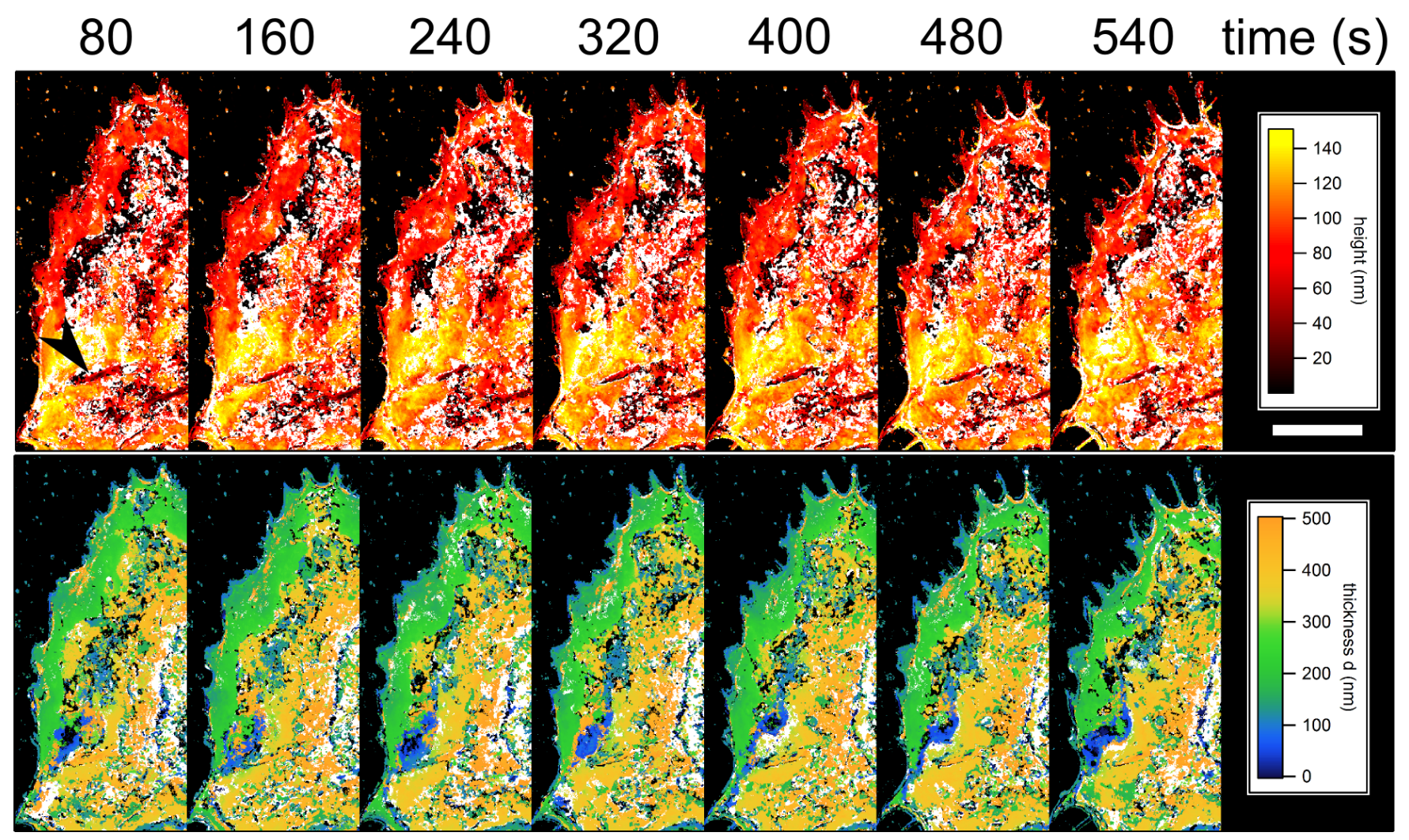

Figure S9. 3D-RICN Reconstruction of $h, d$ (all in $\mathrm{nm}$ ) for a sequence of ca. 10 min long. Scale bar: $10 \mu \mathrm{m}$. Black indicates values under $1 \mathrm{~nm}$, white values above $150 \mathrm{~nm}$ for $h$, or above $500 \mathrm{~nm}$ for $d$. Black arrow on first image of $h$ shows a stress fiber. The lamellipod region is essentially in red for $h$ and in green for $d$. 